\title{
78. Extracellular Localization of Activator Calcium in Dog Coronary Artery Smooth Muscle Fibers
}

\author{
By Haruo SUgI, Suechika SUzukI, and Norihiko FUJIEDA \\ Department of Physiology, School of Medicine, Teikyo University, \\ Itabashi-ku, Tokyo 173
}

(Communicated by Yasuji KAtsuki, M. J. A., Dec. 12, 1981)

In various types of smooth muscle, the pyroantimonate method has been proved to be useful in detecting the intracellular localization of activator $\mathrm{Ca}$ and its translocation during contraction. ${ }^{1-5)}$ In the present work, the pyroantimonate method was applied to examine the localization of activator $\mathrm{Ca}$ in dog coronary artery smooth muscle, and the extracellular origin of activator Ca was demonstrated both physiologically and electron microscopically.

Experiments were performed with small helical strips $(5 \mathrm{~mm}$ long and $1 \mathrm{~mm}$ wide) prepared from isolated dog coronary artery. The preparation was mounted horizontally in a chamber $(3 \mathrm{ml})$ filled with the experimental solution at $37^{\circ} \mathrm{C}$; one end was clamped while the other end was connected to a strain gauge (U- gage, Shinko) to record isometric tension. The steady baseline tension of the preparation was $0.2-0.5 \mathrm{~g}$. The standard solution had the following composition $(\mathrm{mM}): \mathrm{NaCl}, 136.9 ; \mathrm{KCl}, 2.7 ; \mathrm{CaCl}_{2}, 1.8 ; \mathrm{MgCl}_{2}, 1.0 ; \mathrm{NaHCO}_{3}$, $11.9 ; \mathrm{NaHPO}_{4}, 0.4$; glucose, $5.5\left(\mathrm{pH} 7.3\right.$ with $\left.95 \% \mathrm{O}_{2}-5 \% \mathrm{CO}_{2}\right)$. High$\mathrm{K}$ and $\mathrm{Mn}$-containing solutions were made by removing osmotically equivalent amounts of Na. Solutions were changed within 5 sec by a water-vacuum suction tube. For histochemical examination of Ca localization, the preparation was fixed by replacing the experimental solution with $1 \% \mathrm{OsO}_{4}$ solution containing $2 \% \mathrm{~K}$-proantimonate. Further details of the method have been described elsewhere. ${ }^{1)-5)}$

The results of physiological experiments are summarized in Fig. 1. The K-induced contracture tension decreased with a reduction of [Ca $]_{0}$ (Fig. 1A), and eliminated with $20 \mathrm{mM}-\mathrm{Mn}$ (Fig. 1B) which is known to block Ca-influx in smooth muscle. ${ }^{6}$. In Ca-free solution, the steady baseline tension was reduced almost to zero (Fig. 1C), and the preparation no longer showed tension development in response to high $[\mathrm{K}]_{0}$. Verapamil $(1 \mathrm{mM})$, a Ca-antagonist, also showed a similar effect as that of Ca-free solution (Fig. 1D). Noradrenaline $\left(10^{-8}\right.$ $10^{-5} \mathrm{M}$ ) produced relaxation of the baseline tension, but never 

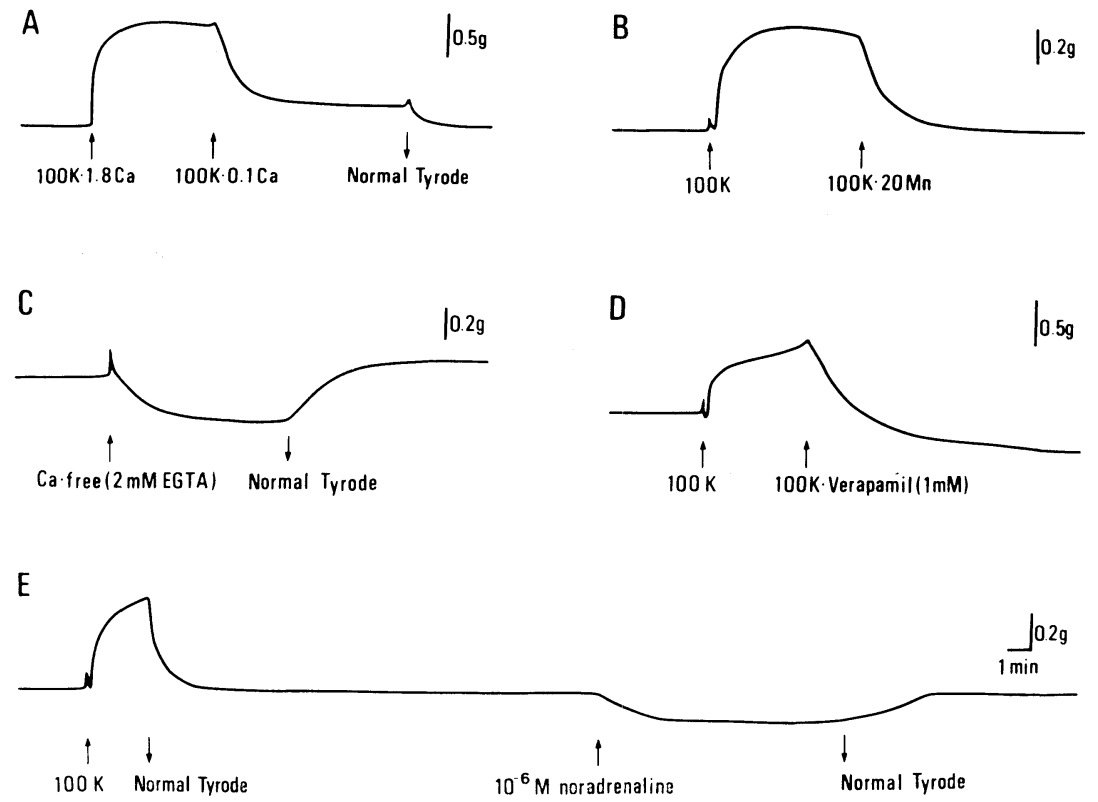

Fig. 1. Tension responses in dog coronary artery smooth muscle fibers. A : Decrease of $\mathrm{K}$-contracture tension by a reduction of [Ca $]_{0}$ from 1.8 to $0.1 \mathrm{M}$. B: Elimination of K-contractura tension by $20 \mathrm{mM}-\mathrm{Mn}$. C: Reduction of baseline tension in Ca-free solution. D: Elimination of $\mathrm{K}$-contracture tension followed by reduction of baseline tension by $1 \mathrm{mM}$ verapamil. $\mathrm{E}$ : Relaxation of baseline tension by $10^{-6} \mathrm{M}$ noradrenaline.

produced tension development (E).. ${ }^{7}$ No appreciable tension could be produced by the factors known to cause Ca release from the intracellular structures in various smooth muscles, such as caffeine $(10 \mathrm{mM}){ }^{8)}$ removal of external divalent cations ${ }^{8)}$ and rapid cooling.9 (10) These results indicate that, in dog coronary artery smooth muscle, activation of the contractile mechanism during K-contractures and the actively maintained baseline tension is associated with the inward movement of extracellular $\mathrm{Ca}$, but not with the release of intracellularly stored Ca. The extracellular origin of activator $\mathrm{Ca}$ in dog coronary artery has also been suggested by van Breemen and Siegel. ${ }^{11)}$

Fig. 2A shows the cross-section of the muscle fibers fixed at the relaxed state. Electron-opaque pyroantimonate precipitate was observed at the nucleus, at the mitochondria and in the lumen of the caveolae, while no marked precipitate was seen along the inner surface of the plasma membrane and at other intracellular structures closely apposed to the plasma membrane. When the fibers were fixed at the contracted state, the precipitate was diffusely distributed in the 


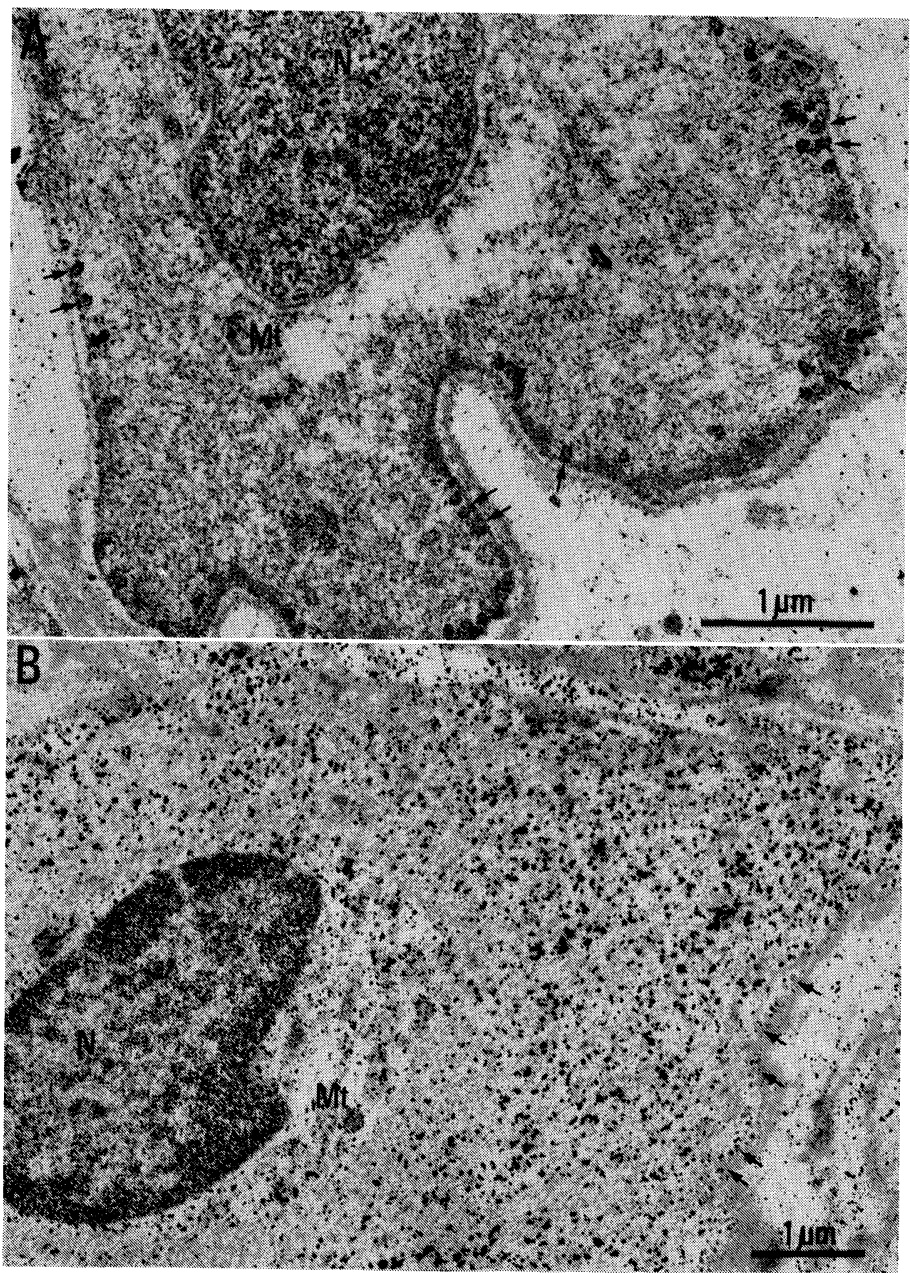

Fig. 2. A : Cross-section of relaxed muscle fibers. To facilitate penetration of pyroantimonate into the fibers, they were kept in an isotonic sucrose solution containing $60 \mathrm{mM} \mathrm{K}$-proantimonate for $10 \mathrm{~min}$ before fixation. Electron-opaque pyroantimonate precipitate can be seen at the nucleus $(\mathrm{N})$, at the mitochondria (Mt) and in the lumen of the caveolae (arrows), which are invaginations of the plasma membrane. B: Cross-section of contracted muscle fibers. They were fixed during a K-contracture produced by $100 \mathrm{mM}-\mathrm{K}$. Note diffuse distribution of the precipitate in the myoplasm in the form of small particles. The precipitate is also seen at the nucleus $(\mathrm{N})$ and the mitochondria (M). Also note the decrease in the amount of precipitate in the caveolae lumen (arrows). In both $\mathbf{A}$ and $\mathrm{B}$, the section was stained with uranyl acetate and lead citrate.

myoplasm in the form of small particles, and the precipitate in the caveolar lumen was no longer observable, whereas the amount of the precipitate at the nucleus and the mitochondria appeared to remain unchanged (Fig. 2B). Electron-probe X-ray microanalysis of the 
precipitate in both relaxed and contracted fibers ${ }^{2)-5)}$ always showed the presence of $\mathrm{Ca}$, indicating that the pyroantimonate precipitate serves as a valid measure of $\mathrm{Ca}$ localization.

Since caveolae are bottle-shaped invaginations of the plasma membrane, ${ }^{12)}$ their lumen is continuous with the extracellular space. Thus, the presence of the precipitate in the caveolae lumen in the relaxed fibers, together with the absence of the marked intracellular precipitate in the vicinity of the plasma membrane, strongly suggest that dog coronary artery smooth muscle fibers do not contain intracellularly stored activator $\mathrm{Ca}$, and that activator $\mathrm{Ca}$ is localized at least partly in the caveolar lumen as well as other extracellular space. This is completely in accord with the results of physiological experiments that activator $\mathrm{Ca}$ comes from the extracellular space.

We wish to thank Prof. A. Kamiyama for providing the experimental material and for Miss S. Gomi for her technical assistance.

\section{References}

1) Atsumi, S., and Sugi, H.: J. Physiol., 257, 549-560 (1976).

2) Sugi, H., and Daimon, T.: Nature, 269, 436-438 (1977).

3) Suzuki, S., and Sugi, H.: J. Cell Biol., 79, 467-478 (1978).

4) Sugi, H., Suzuki, S., and Daimon, T.: Canad. J. Physiol. Pharmacol. (in press).

5) Suzuki, S., and Sugi, H.: J. Exp. Biol. (in press).

6) Nonomura, Y., Hotta, Y., and Ohashi, H.: Science, 152, 97-99 (1966).

7) Toda, N.: Biomed. Ther., 4, 14-19 (1980).

8) Sugi, H., and Yamaguchi, T.: J. Physiol., 257, 531-547 (1976).

9) Sugi, H., and Suzuki, S.: J. Cell Biol., 79, 454-466 (1978).

10) Sakai, T., and Kurihara, S.: Jikeikai Med. J., 21, 47-88 (1974).

11) van Breemen, C., and Siegel, B.: Circ. Res., 46, 426-429 (1980).

12) Forbes, M. S., Rennels, M. L., and Nelson, E.: J. Ultrastruct. Res., 67, 325-339 (1979). 